\title{
An Update on the Role of the Actin Cytoskeleton in Plasmodesmata: A Focus on Formins
}

\section{OPEN ACCESS}

Edited by:

Jung-Youn Lee,

University of Delaware, United States

Reviewed by:

Viktor Zarsky,

Charles University, Czechia

Rosemary White,

Commonwealth Scientific and Industrial Research Organisation

(CSIRO), Australia

*Correspondence:

Shanjin Huang

sjhuang@tsinghua.edu.cn

Specialty section: This article was submitted to Plant Cell Biology, a section of the journal

Frontiers in Plant Science

Received: 29 December 2020

Accepted: 26 January 2021

Published: 15 February 2021

Citation:

Diao M and Huang S (2021) An Update on the Role of the Actin

Cytoskeleton in Plasmodesmata: $A$

Focus on Formins.

Front. Plant Sci. 12:647123.

doi: 10.3389/fp/s.2021.647123

\author{
Min Diao ${ }^{2}$ and Shanjin Huang ${ }^{1 *}$ \\ ${ }^{1}$ Center for Plant Biology, School of Life Sciences, Tsinghua University, Beijing, China, ${ }^{2}$ iHuman Institute, Shanghai Tech \\ University, Shanghai, China
}

Cell-to-cell communication in plants is mediated by plasmodesmata (PD) whose permeability is tightly regulated during plant growth and development. The actin cytoskeleton has been implicated in regulating the permeability of $\mathrm{PD}$, but the underlying mechanism remains largely unknown. Recent characterization of PD-localized formin proteins has shed light on the role and mechanism of action of actin in regulating PD-mediated intercellular trafficking. In this mini-review article, we will describe the progress in this area.

Keywords: intercellular trafficking, plasmodesmata, actin, actin-binding protein, formin

\section{INTRODUCTION}

The growth and development of multicellular organisms requires intercellular communication. Intercellular communication in plants can be classified into symplasmic and apoplasmic pathways. For the symplasmic pathway, intercellular communication is achieved through complex channellike structures embedded within the cell walls, called plasmodesmata (PD). The development of the PD structure enables the trafficking of molecules between adjacent plant cells, including some small molecules, such as ions, carbohydrates, and hormones, as well as some large molecules including RNAs, proteins, and viruses (Tilsner et al., 2016; Lee and Frank, 2018). As such, PD are involved in the regulation of plant growth and development and environmental adaptation including disease resistance (Cheval and Faulkner, 2018). The structure and function of PD must be tightly regulated throughout the life of a plant (Lee and Frank, 2018). Indeed, many factors have been shown to be involved in regulating the permeability of PD. For instance, the callose at the neck of $\mathrm{PD}$ is involved in the regulation of intercellular trafficking in plants. It was shown that callose deposition at PD will accelerate during virus infection in order to prevent the spread of viruses (Levy et al., 2007). In line with this finding, some viruses have movement proteins (MPs), which can mediate the degradation of callose to open up PD (Schoelz et al., 2011). In addition, consistent with the presence of actin cytoskeletal proteins in $\mathrm{PD}$, the actin cytoskeleton has been implicated in the regulation of intercellular trafficking via PD (White and Barton, 2011; Pitzalis and Heinlein, 2017), but the underlying mechanism remains largely unexplored. In this mini-review, we are going to describe the recent progress made in this respect. 


\section{EVIDENCE SUPPORTING THE ROLE OF ACTIN IN REGULATING THE PERMEABILITY OF PD}

Actin is a highly conserved $42 \mathrm{kDa}$ protein, and it is very abundant in eukaryotes. Actin is involved in many cellular physiological processes in plants, including cell growth, cell division, cytokinesis, and various intracellular trafficking events. As such, actin plays a crucial role in plant growth and development (Szymanski and Staiger, 2018). Under optimal conditions, actin can assemble into filamentous structures, called actin filaments (F-actin) or microfilaments. Most actin-based functions are dictated by the spatial organization and dynamics of F-actin in cells. Within cells, actin is associated with many proteins, called actin-binding proteins ( $\mathrm{ABP}$ ), which modulate the kinetics of actin assembly and disassembly as well as facilitating the formation of different actin structures (Wang et al., 2015). Characterization of the role and mechanism of action of ABPs promises to provide insights into the action of actin within different cellular physiological processes.

Experimental treatments with actin-based pharmacological agents showed that the actin cytoskeleton is involved in the regulation of intercellular communication via PD. It was shown that the transport efficiency through PD increases after microinjection of specific actin depolymerizers into tobacco mesophyll cells, whereas the transport efficiency decreases after microinjection of the microfilament stabilizer phalloidin into the cells (Ding et al., 1996; Su et al., 2010). In line with these findings, treatment with the myosin inhibitor 2,3-butanedione monoxime (BDM) reduces the neck width of PD (Radford and White, 1998). However, given that those drugs non-selectively target the actin cytoskeletal system within cells, it remains uncertain whether, and to what extent, the changes in structure and function of PD result from the alteration in the actin cytoskeletal system.

In addition to functioning in plant growth and development, $\mathrm{PD}$ are involved in defense against plant pathogens (Cheval and Faulkner, 2018). The important role of PD in virus infection is quite obvious, as viruses spread between cells using PD as the channels. Plant viruses encode MPs to mediate the intercellular transport of infectious genomes via PD. It was reported that MPs can mediate the degradation of callose to open up PD (Schoelz et al., 2011). Besides that, another interesting report showed that MPs open up PD via interacting with the actin cytoskeleton in PD. Specifically, it was shown that Cucumber mosaic virus (CMV) MP severs and caps actin filaments in vitro and its filament severing activity is required for its function in PD (Su et al., 2010). Accordingly, it was shown that pretreatment with the actin monomer sequestering reagent latrunculin A (LatA) to depolymerize actin filaments promotes the function of MP in opening up PD, whereas pretreatment with phalloidin to stabilize actin filaments has the opposite effect (Su et al., 2010). These studies imply that there might exist endogenous ABPs that are involved in regulating the permeability of PD via controlling actin dynamics in PD. However, due to the lack of techniques to directly visualize the actin cytoskeleton in PD, there is still a debate about whether filamentous actin exists in PD and, if so, how it is organized. This prevents us from understanding the function of the actin cytoskeleton in regulating cell-to-cell trafficking via $\mathrm{PD}$. In this regard, development of technology enabling the visualization of the actin cytoskeleton in PD is extremely necessary. In addition, development of methods to specifically alter actin dynamics in PD might provide insights into the function and mechanism of action of actin in the regulation of PD function.

\section{THE PRESENCE OF ACTIN AND ACTIN- BINDING PROTEINS IN PD}

The involvement of the actin cytoskeleton in regulating the function of PD is also supported by data showing that actin and some ABPs associate with PD. The association of actin with PD was initially discovered by the immunogold labeling approach (Table 1; White et al., 1994; Blackman and Overall, 1998) using a monoclonal antibody against chicken gizzard actin. The association of actin with PD structures was further confirmed using fluorescent phalloidins or by immunofluorescence using an antibody against human actin (Table 1; Baluska et al., 2001, 2004).

Similarly, myosin was first discovered to associate with PD with immuno-EM using polyclonal antibodies against animal myosins (Table 1; Blackman and Overall, 1998; Radford and White, 1998), which recognize highly conserved motifs in the myosin head, as well as an antibody against the C-terminal tail of plant myosin VIII (Table 1; Reichelt et al., 1999). The association of myosins with PD was also verified by immunofluorescence analyses with the same antibodies (Table 1; Radford and White, 1998; Reichelt et al., 1999; Baluska et al., 2001, 2004). Subsequent analysis of myosin XI fused to different fluorescent proteins

TABLE 1 | Actin and its associated proteins identified in plasmodesmata (PD).

\begin{tabular}{|c|c|c|}
\hline $\begin{array}{l}\text { Cytoskeletal } \\
\text { protein }\end{array}$ & Function & Reference(s) \\
\hline Actin & $\begin{array}{l}\text { Building blocks of the } \\
\text { actin cytoskeleton }\end{array}$ & $\begin{array}{l}\text { White et al., 1994; Ding et al., 1996; } \\
\text { Blackman and Overall, 1998; } \\
\text { Fernandez-Calvino et al., } 2011\end{array}$ \\
\hline Myosin & $\begin{array}{l}\text { Actin filament side } \\
\text { binding; actin-based } \\
\text { movement }\end{array}$ & $\begin{array}{l}\text { Blackman and Overall, 1998; Radford } \\
\text { and White, 1998; Reichelt et al., 1999; } \\
\text { Volkmann et al., 2003; Wojtaszek et al., } \\
\text { 2005; Golomb et al., 2008; Sattarzadeh } \\
\text { et al., 2008; Fernandez-Calvino et al., } \\
\text { 2011; Haraguchi et al., } 2014\end{array}$ \\
\hline Tropomyosin & $\begin{array}{l}\text { Actin filament side } \\
\text { binding }\end{array}$ & $\begin{array}{l}\text { Faulkner et al., 2009; Fernandez- } \\
\text { Calvino et al., } 2011\end{array}$ \\
\hline ARP2/3 & Actin nucleation & Van Gestel et al., 2003 \\
\hline NET & Actin binding & Deeks et al., 2012 \\
\hline Formin & $\begin{array}{l}\text { Barbed end capping, } \\
\text { actin nucleation }\end{array}$ & Diao et al., 2018; Oulehlova et al., 2019 \\
\hline Profilin & Actin monomer binding & Fernandez-Calvino et al., 2011 \\
\hline ADF & $\begin{array}{l}\text { Actin filament severing; } \\
\text { actin monomer binding }\end{array}$ & Fernandez-Calvino et al., 2011 \\
\hline GSD1 & Actin binding & Gui et al., 2014 \\
\hline
\end{tabular}


showed no localization to PD (Reisen and Hanson, 2007). Interestingly, one GFP fusion with the IQ-tail zone of ATM1, a member of the Arabidopsis myosin VIII family, appears to localize to sites of ER attachment as well as pitfields when expressed in Nicotiana benthamiana leaves (Golomb et al., 2008).

In addition, it was shown that tropomyosin-like proteins localize to PD and cell plates using antibodies against mammalian tropomyosins (Table 1; Faulkner et al., 2009). Using the same approach, it was shown that actin-related protein 3 (Arp3) is localized in PD and multivesicular bodies (MVBs) in maize and tobacco (Table 1; Van Gestel et al., 2003). In addition, it was shown that a plant-specific ABP, network protein $1 \mathrm{~A}$ (NET1A), is able to localize to PD (Table 1; Deeks et al., 2012). Another interesting report showed that grain setting defect1 (GSD1), a plant-specific remorin protein, is able to interact with actin (Gui et al., 2015) and can localize to PD (Table 1; Gui et al., 2014). The presence of ABPs in PD was also supported by data showing that profilin and ADF are present in the Arabidopsis plasmodesmal proteome (Table 1; Fernandez-Calvino et al., 2011). Certainly, direct cytological evidence is needed to confirm that these proteins are indeed localized to PD. Interestingly, recent characterization showed that several Arabidopsis and rice class I formins associate with PD (Table 1; Diao et al., 2018; Oulehlova et al., 2019). In summary, actin and some ABPs are able to associate with PD.

\section{THE ROLE OF CLASS I FORMINS IN REGULATING THE PERMEABILITY OF PD}

Formin (formin homology protein) nucleates actin assembly for the generation of linear actin bundles. The formin proteins contain the characteristic formin homology domain 1 (FH1) and $\mathrm{FH} 2$, which are capable of nucleating actin assembly from actin or actin-profilin complexes. The biochemical activities of plant formins have been characterized extensively in vitro and most of them are typical formins that nucleate actin assembly from actin or actin bound to profilin (van Gisbergen and Bezanilla, 2013). In vitro biochemical analysis revealed that some plant formins have evolved some unusual activities. For instance, AtFH1 was shown to be a nonprocessive actin polymerase, which can bundle actin filaments (Michelot et al., 2006). The formin proteins have been implicated in numerous actin-based cellular processes in plants, such as pollen germination (Lan et al., 2018; Liu et al., 2018), polarized pollen tube growth and root hair growth (Ye et al., 2009; Cheung et al., 2010; Huang et al., 2013; Lan et al., 2018), cell division (Li et al., 2010), cytokinesis (Ingouff et al., 2005), and cell expansion (Yang et al., 2011; Zhang et al., 2011), as well as defense (Favery et al., 2004). There are 21 formin genes in the Arabidopsis genome, and the encoded proteins can be divided into two classes (Blanchoin and Staiger, 2010). Specifically, there are 11 class I formins and 10 class II formins in Arabidopsis. Among them, Class I formins contain the characteristic transmembrane domain (TMD) at their N-terminus, which enable them to target to membranes (van Gisbergen and Bezanilla, 2013).
Interestingly, recent studies showed that several class I formins specifically localize to PD (Diao et al., 2018; Oulehlova et al., 2019) and they are involved in regulating the permeability of PD in Arabidopsis (Diao et al., 2018). It was shown that the class I formin AtFH2 localizes to PD in various tissues, and this function is dictated by its N-terminal TMD. Analysis of atfh 2 mutants showed that the permeability of PD is increased when compared to WT. As such, atfh2 mutants are sensitive to virus infection. Strikingly, it was shown that a mutant AtFH2, which was deficient in interacting with actin filaments, failed to rescue the defective intercellular trafficking via $\mathrm{PD}$ in atfh2 mutants. This suggests that the interaction of AtFH2 with the actin cytoskeleton is crucial for its function in $\mathrm{PD}$. In vitro biochemical analysis showed that AtFH2 lacks actin nucleation activity but it caps the barbed end of actin filaments and stabilizes them against dilution-mediated depolymerization in vitro (Diao et al., 2018). This allows us to speculate that actin filaments become instable and/or the amount of actin filaments is reduced in $\mathrm{PD}$ in atfh 2 mutants. It is quite unusual that AtFH2 can cap the barbed end of actin filaments to prevent their elongation but fails to nucleate actin assembly in vitro. Certainly, it cannot be completely ruled out that AtFH2 is able to nucleate actin assembly after post-translational modification or by interacting with some partners in vivo. Nonetheless, the current in vitro biochemical data suggest that AtFH2 regulates actin dynamics only by binding to the barbed end of filamentous actin. To some extent, this supports the notion that actin filaments exist in PD. Certainly, uncovering the precise localization of AtFH2 in $\mathrm{PD}$ will further refine this hypothesis. However, we still do not know how to fit actin filaments into PD as the gap between the plasma membrane and the ER (called the cytoplasmic sleeve) within PD pores is less than $10 \mathrm{~nm}$ (Nicolas et al., 2017). It could be possible that actin filaments stay in cytoplasmic sleeve but twine around the ER within PD pores. In addition, Nicolas et al. (2017) also discovered a second PD morphotype (type I) that lacks a visible cytoplasmic sleeve but is capable of non-targeted movement of macromolecules, which indicates that the size of PD pores undergoes dynamic changes. Therefore, the space of cytoplasmic sleeve might increase substantially under certain condition that allows the fitting of actin filaments.

Interestingly, it was shown that several other class I formins are also able to target to PD. Specifically, the closest homolog of AtFH2, namely AtFH1, is also able to associate with PD (Diao et al., 2018; Oulehlova et al., 2019). AtFH1 functions redundantly with $\mathrm{AtFH} 2$ in regulating the permeability of $\mathrm{PD}$ (Diao et al., 2018). Strikingly, it was shown that several rice class I formins are also able to target to PD (Diao et al., 2018), suggesting that targeting of class I formins to PD is an evolutionarily conserved strategy in plants. An interesting but yet-to-be-answered question is how the TMD of the PD-localized class I formins have evolved to enable their targeting to PD. This function may be linked to the fact that the membrane of $\mathrm{PD}$ has a unique phospholipid composition (Grison et al., 2015). Certainly, it could be possible that the TMD of those class I formins might have additional functions besides the membrane anchoring. In support of this speculation, a very recent report showed that 


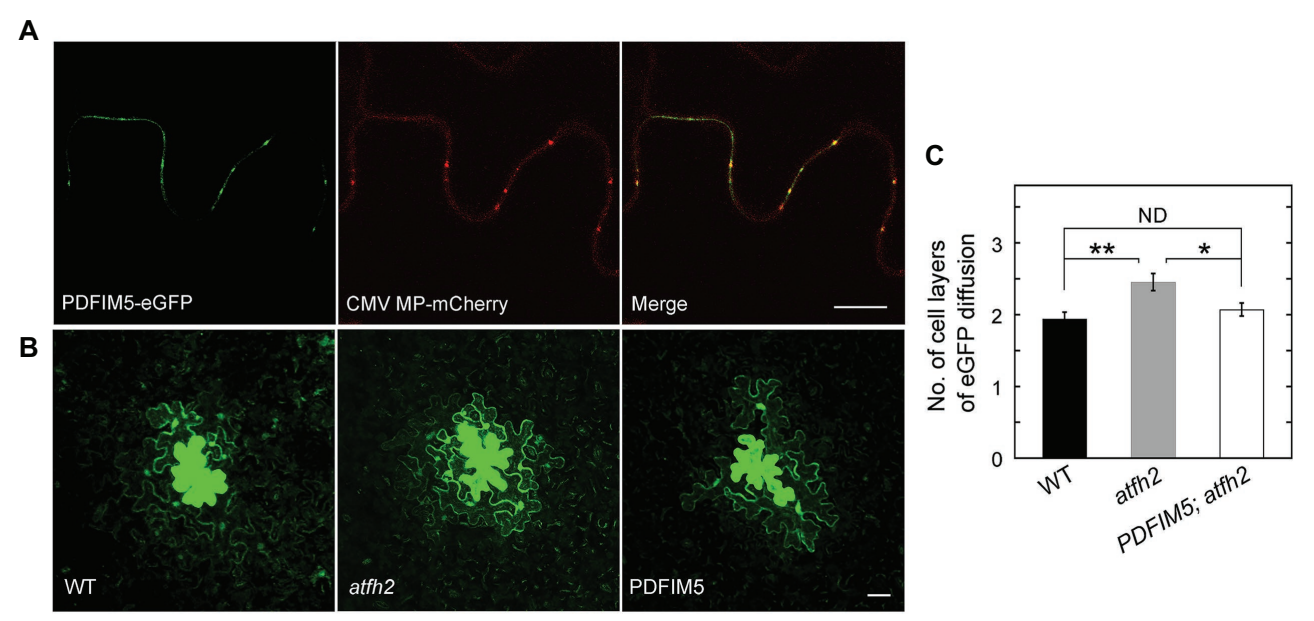

FIGURE 1 | Targeting of FIMBRIN 5 (FIM5) to PD Alleviates the PD Phenotype in atfh2 Mutants. (A) Subcellular localization of PDFIM5-eGFP and Cucumber mosaic virus (CMV) movement protein (MP)-mCherry in epidermal pavement cells of Nicotiana benthamiana leaves. PDFIM5 was obtained by fusing the N-terminal fragment of AtFH2 (AtFH2 ${ }^{\text {N282 }}$ ) with Arabidopsis FIMBRIN5 (Wu et al., 2010). PDFIM5 was further fused to eGFP (Diao et al., 2018). Plasmids encoding PDFIM5eGFP and CMV MP-mCherry were introduced into Agrobacterium tumefaciens strain GV3101 and transiently expressed in N. benthamiana leaves by GV3101 injection. Bar $=10 \mu \mathrm{m}$. (B) Images of eGFP diffusion in leaf epidermal pavement cells of WT, atfh2, and PDFIM5; atfh2 plants. PDFIM5 was constructed as in (A). The PDFIM5 plasmid was introduced into Agrobacterium tumefaciens strain GV3101 and transformed into atfh2 plants by the floral dip method. The PD permeability of WT, atfh2, and atfh2 harboring PDFIM5 was assessed by the eGFP diffusion assay (Diao et al., 2019), and the images were collected by confocal microscopy. Bar $=10 \mu \mathrm{m}$. (C) Quantification of the number of cell layers with eGFP diffusion in Arabidopsis leaf epidermal pavement cells at 24 h after bombardment. PDFIM5; atfh2 represents atfh2 plants expressing PDFIM5. More than 30 cells were counted and the experiments were repeated at least three times. Error bars represent SE. ${ }^{*} p<0.05$ and ${ }^{* *} p<0.01$ by Mann-Whitney $\cup$ test. ND, no statistical difference.

TMD of Arabidopsis thaliana Plasmodesmata-located protein (PDLP) 5 is involved in the self-interaction of PDLP5 that is essential for PDLP5 to regulate cell-to-cell movement besides its role in membrane targeting (Wang et al., 2020).

As mentioned above, $\mathrm{PD}$ permeability is increased in atfh2 mutants. Interestingly, targeting of Arabidopsis FIMBRIN 5 (FIM5) to PD alleviates the intercellular trafficking phenotype in atfh2 mutants (Figure 1). This suggests that loss of AtFH2 causes instability of actin filaments and/or reduction in the amount of actin filaments in PD. These data actually support the previous notion that stabilization of actin filaments decreases the permeability of PD whereas destabilization of actin filaments increases it (Ding et al., 1996; Su et al., 2010). In summary, these data together suggest that the amount of actin filaments and/or the stability of actin filaments are crucial for the permeability of PD, and actin filaments in $\mathrm{PD}$ presumably act as the physical barrier to regulate the permeability of PD.

\section{CONCLUSIONS AND PERSPECTIVES}

Increasing evidence is showing that the actin cytoskeleton is involved in the regulation of intercellular transport through $\mathrm{PD}$, whereas the molecular mechanism by which the actin cytoskeleton regulates the permeability of $\mathrm{PD}$ remains largely unexplored. Research in this area progresses slowly for at least two reasons. Firstly, researchers lack approaches to directly visualize the actin cytoskeleton in $\mathrm{PD}$, because $\mathrm{PD}$ are tiny structures that are deeply embedded in the cell walls. Secondly, researchers lack approaches to specifically manipulate the function of the actin cytoskeleton in PD. Recent identification of PD-localized class I formins provides the possibility to manipulate the actin cytoskeleton in $\mathrm{PD}$ via regulating the function of those formins. Indeed, analysis of PD permeability in mutants lacking AtFH2 or AtFH1 and AtFH2, in combination with in vitro biochemical characterization of AtFH2, allows us to conclude that actin filaments might act as the physical barrier in controlling the permeability of PD. This is actually consistent with a previous assumption that actin filaments in PD might act as the filter in controlling PD permeability (Chen et al., 2010). However, the precise localization of AtFH2 in PD is currently unknown. Dissection of the AtFH2mediated actin regulatory machinery in $\mathrm{PD}$, for example, by searching for AtFH2-interacting proteins or screening for suppressors or enhancers of the atfh 2 mutant phenotype, might provide further insights into the function and regulation of the actin cytoskeleton in PD. In summary, recent characterizations of PD-localized class I formins have provided insights into the function and mechanism of action of actin in regulating the permeability of PD. However, it remains largely unknown how exactly the actin cytoskeleton regulates the structure and function of PD. This will be an exciting research avenue in the future.

\section{AUTHOR CONTRIBUTIONS}

All authors listed have made a substantial, direct and intellectual contribution to the work, and approved it for publication. 


\section{FUNDING}

This work was supported by a grant from National Natural Science Foundation of China (31970180) and the funding from Beijing Advanced Innovation Center for Structural Biology.

\section{REFERENCES}

Baluska, F., Cvrckova, F., Kendrick-Jones, J., and Volkmann, D. (2001). Sink plasmodesmata as gateways for phloem unloading. Myosin VIII and calreticulin as molecular determinants of sink strength? Plant Physiol. 126, 39-46. doi: 10.1104/pp.126.1.39

Baluska, F., Samaj, J., Hlavacka, A., Kendrick-Jones, J., and Volkmann, D. (2004). Actin-dependent fluid-phase endocytosis in inner cortex cells of maize root apices. J. Exp. Bot. 55, 463-473. doi: 10.1093/jxb/erh042

Blackman, L. M., and Overall, R. L. (1998). Immunolocalisation of the cytoskeleton to plasmodesmata of Chara corallina. Plant J. 14, 733-741. doi: 10.1046/j. 1365-313x.1998.00161.x

Blanchoin, L., and Staiger, C. J. (2010). Plant formins: diverse isoforms and unique molecular mechanism. Biochim. Biophys. Acta 1803, 201-206. doi: 10.1016/j.bbamcr.2008.09.015

Chen, C., Zhang, Y., Zhu, R., and Yuan, M. (2010). The actin cytoskeleton is involved in the regulation of the plasmodesmal size exclusion limit. Plant Signal. Behav. 5, 1663-1665. doi: 10.4161/psb.5.12.14018

Cheung, A. Y., Niroomand, S., Zou, Y., and Wu, H. M. (2010). A transmembrane formin nucleates subapical actin assembly and controls tip-focused growth in pollen tubes. Proc. Natl. Acad. Sci. U. S. A. 107, 16390-16395. doi: 10.1073/ pnas. 1008527107

Cheval, C., and Faulkner, C. (2018). Plasmodesmal regulation during plantpathogen interactions. New Phytol. 217, 62-67. doi: 10.1111/nph.14857

Deeks, M. J., Calcutt, J. R., Ingle, E. K., Hawkins, T. J., Chapman, S., Richardson, A. C., et al. (2012). A superfamily of actin-binding proteins at the actin-membrane nexus of higher plants. Curr. Biol. 22, 1595-1600. doi: 10.1016/j.cub.2012.06.041

Diao, M., Ren, S., Wang, Q., Qian, L., Shen, J., Liu, Y., et al. (2018). Arabidopsis formin 2 regulates cell-to-cell trafficking by capping and stabilizing actin filaments at plasmodesmata. Elife 7:e36316. doi: 10.7554/eLife.36316

Diao, M., Wang, Q. N., and Huang, S. J. (2019). Quantitative plasmodesmata permeability assay for pavement cells of Arabidopsis leaves. Bio-Protocol 9:e3206. doi: 10.21769/BioProtoc.3206

Ding, B., Kwon, M. O., and Warnberg, L. (1996). Evidence that actin filaments are involved in controlling the permeability of plasmodesmata in tobacco mesophyll. Plant J. 10, 157-164. doi: 10.1046/j.1365-313X.1996.100 10157.x

Faulkner, C. R., Blackman, L. M., Collings, D. A., Cordwell, S. J., and Overall, R. L. (2009). Anti-tropomyosin antibodies co-localise with actin microfilaments and label plasmodesmata. Eur. J. Cell Biol. 88, 357-369. doi: 10.1016/j. ejcb.2009.02.184

Favery, B., Chelysheva, L. A., Lebris, M., Jammes, F., Marmagne, A., De Almeida-Engler, J., et al. (2004). Arabidopsis formin AtFH6 is a plasma membrane-associated protein upregulated in giant cells induced by parasitic nematodes. Plant Cell 16, 2529-2540. doi: 10.1105/tpc.104.024372

Fernandez-Calvino, L., Faulkner, C., Walshaw, J., Saalbach, G., Bayer, E., Benitez-Alfonso, Y., et al. (2011). Arabidopsis plasmodesmal proteome. PLoS One 6:e18880. doi: 10.1371/journal.pone.0018880

Golomb, L., Abu-Abied, M., Belausov, E., and Sadot, E. (2008). Different subcellular localizations and functions of Arabidopsis myosin VIII. BMC Plant Biol. 8:3. doi: 10.1186/1471-2229-8-3

Grison, M. S., Brocard, L., Fouillen, L., Nicolas, W., Wewer, V., Dormann, P., et al. (2015). Specific membrane lipid composition is important for plasmodesmata function in Arabidopsis. Plant Cell 27, 1228-1250. doi: 10.1105/ tpc.114.135731

Gui, J. S., Liu, C., Shen, J. H., and Li, L. G. (2014). Grain setting defect1, encoding a remorin protein, affects the grain setting in rice through regulating plasmodesmatal conductance. Plant Physiol. 166, 1463-1478. doi: 10.1104/ pp.114.246769

\section{ACKNOWLEDGMENTS}

We thank the members of the Huang lab for helpful discussion on this review. We apologize to those whose work could not be cited due to space limitations.

Gui, J. S., Zheng, S., Shen, J. H., and Li, L. G. (2015). Grain setting defectl (GSD1) function in rice depends on S-acylation and interacts with actin 1 (OsACT1) at its C-terminal. Front. Plant Sci. 6:804. doi: 10.3389/ fpls.2015.00804

Haraguchi, T., Tominaga, M., Matsumoto, R., Sato, K., Nakano, A., Yamamoto, K., et al. (2014). Molecular characterization and subcellular localization of Arabidopsis class VIII myosin, ATM1. J. Biol. Chem. 289, 12343-12355. doi: 10.1074/jbc.M113.521716

Huang, J., Kim, C. M., Xuan, Y. H., Liu, J., Kim, T. H., Kim, B. K., et al. (2013). Formin homology 1 (OsFH1) regulates root-hair elongation in rice (Oryza sativa). Planta 237, 1227-1239. doi: 10.1007/s00425-013-1838-8

Ingouff, M., Fitz Gerald, J. N., Guerin, C., Robert, H., Sorensen, M. B., Van Damme, D., et al. (2005). Plant formin AtFH5 is an evolutionarily conserved actin nucleator involved in cytokinesis. Nat. Cell Biol. 7, 374-380. doi: $10.1038 /$ ncb 1238

Lan, Y., Liu, X., Fu, Y., and Huang, S. (2018). Arabidopsis class I formins control membrane-originated actin polymerization at pollen tube tips. PLoS Genet. 14:e1007789. doi: 10.1371/journal.pgen.1007789

Lee, J. Y., and Frank, M. (2018). Plasmodesmata in phloem: different gateways for different cargoes. Curr. Opin. Plant Biol. 43, 119-124. doi: 10.1016/j. pbi.2018.04.014

Levy, A., Erlanger, M., Rosenthal, M., and Epel, B. L. (2007). A plasmodesmataassociated beta-1,3-glucanase in Arabidopsis. Plant J. 49, 669-682. doi: 10.1111/j.1365-313X.2006.02986.x

Li, Y., Shen, Y., Cai, C., Zhong, C., Zhu, L., Yuan, M., et al. (2010). The type II Arabidopsis formin14 interacts with microtubules and microfilaments to regulate cell division. Plant Cell 22, 2710-2726. doi: 10.1105/tpc.110.075507

Liu, C., Zhang, Y., and Ren, H. (2018). Actin polymerization mediated by AtFH5 directs the polarity establishment and vesicle trafficking for pollen germination in Arabidopsis. Mol. Plant 11, 1389-1399. doi: 10.1016/j. molp.2018.09.004

Michelot, A., Derivery, E., Paterski-Boujemaa, R., Guerin, C., Huang, S., Parcy, F., et al. (2006). A novel mechanism for the formation of actin-filament bundles by a nonprocessive formin. Curr. Biol. 16, 1924-1930. doi: 10.1016/j. cub.2006.07.054

Nicolas, W. J., Grison, M. S., Trepout, S., Gaston, A., Fouche, M., Cordelieres, F. P., et al. (2017). Architecture and permeability of post-cytokinesis plasmodesmata lacking cytoplasmic sleeves. Nat. Plants 3:17082. doi: 10.1038/nplants.2017.82

Oulehlova, D., Kollarova, E., Cifrova, P., Pejchar, P., Zarsky, V., and Cvrckova, F (2019). Arabidopsis class I formin FH1 relocates between membrane compartments during root cell ontogeny and associates with plasmodesmata. Plant Cell Physiol. 60, 1855-1870. doi: 10.1093/pcp/pcz102

Pitzalis, N., and Heinlein, M. (2017). The roles of membranes and associated cytoskeleton in plant virus replication and cell-to-cell movement. J. Exp. Bot. 69, 117-132. doi: 10.1093/jxb/erx334

Radford, J. E., and White, R. G. (1998). Localization of a myosin-like protein to plasmodesmata. Plant J. 14, 743-750. doi: 10.1046/j.1365-313x.1998.00162.x

Reichelt, S., Knight, A. E., Hodge, T. P., Baluska, F., Samaj, J., Volkmann, D., et al. (1999). Characterization of the unconventional myosin VIII in plant cells and its localization at the post-cytokinetic cell wall. Plant J. 19, 555-567. doi: 10.1046/j.1365-313X.1999.00553.x

Reisen, D., and Hanson, M. R. (2007). Association of six YFP-myosin XI-tail fusions with mobile plant cell organelles. BMC Plant Biol. 7:6. doi: 10.1186/1471-2229-7-6

Sattarzadeh, A., Franzen, R., and Schmelzer, E. (2008). The Arabidopsis class VIII myosin ATM2 is involved in endocytosis. Cell Motil. Cytoskel. 65, 457-468. doi: $10.1002 / \mathrm{cm} .20271$

Schoelz, J. E., Harries, P. A., and Nelson, R. S. (2011). Intracellular transport of plant viruses: finding the door out of the cell. Mol. Plant 4, 813-831. doi: $10.1093 / \mathrm{mp} / \mathrm{ssr} 070$ 
Su, S., Liu, Z., Chen, C., Zhang, Y., Wang, X., Zhu, L., et al. (2010). Cucumber mosaic virus movement protein severs actin filaments to increase the plasmodesmal size exclusion limit in tobacco. Plant Cell 22, 1373-1387. doi: $10.1105 /$ tpc.108.064212

Szymanski, D., and Staiger, C. J. (2018). The actin cytoskeleton: functional arrays for cytoplasmic organization and cell shape control. Plant Physiol. 176, 106-118. doi: 10.1104/pp.17.01519

Tilsner, J., Nicolas, W., Rosado, A., and Bayer, E. M. (2016). Staying tight: plasmodesmal membrane contact sites and the control of cell-to-cell connectivity in plants. Annu. Rev. Plant Biol. 67, 337-364. doi: 10.1146/annurevarplant-043015-111840

Van Gestel, K., Slegers, H., Von Witsch, M., Samaj, J., Baluska, F., and Verbelen, J. P. (2003). Immunological evidence for the presence of plant homologues of the actin- related protein Arp3 in tobacco and maize: subcellular localization to actin-enriched pit fields and emerging root hairs. Protoplasma 222, 45-52. doi: 10.1007/s00709-003-0004-8

van Gisbergen, P. A., and Bezanilla, M. (2013). Plant formins: membrane anchors for actin polymerization. Trends Cell Biol. 23, 227-233. doi: 10.1016/j. tcb.2012.12.001

Volkmann, D., Mori, T., Tirlapur, U. K., Konig, K., Fujiwara, T., Kendrick-Jones, J., et al. (2003). Unconventional myosins of the plant-specific class VIII: endocytosis, cytokinesis, plasmodesmata/pit-fields, and cell-to-cell coupling. Cell Biol. Int. 27, 289-291. doi: 10.1016/S1065-6995(02)00330-X

Wang, X., Luna, G. R., Arighi, C. N., and Lee, J. Y. (2020). An evolutionarily conserved motif is required for plasmodesmata-located protein 5 to regulate cell-to-cell movement. Commun. Biol. 3:291. doi: 10.1038/s42003-020-1007-0

Wang, J., Zhang, R., Chang, M., Qu, X., Diao, M., Zhang, M., et al. (2015). "Actin cytoskeleton" in The plant sciences-cell biology. Vol. 20. eds. S. Assmann and B. Liu (New York: Springer), 1-28.

White, R. G., Badelt, K., Overall, R. L., and Vesk, M. (1994). Actin associated with plasmodesmata. Protoplasma 180, 169-184. doi: 10.1007/BF01507853
White, R. G., and Barton, D. A. (2011). The cytoskeleton in plasmodesmata: a role in intercellular transport? J. Exp. Bot. 62, 5249-5266. doi: 10.1093/ jxb/err227

Wojtaszek, P., Anielska-Mazur, A., Gabrys, H., Baluska, F., and Volkmann, D. (2005). Recruitment of myosin VIII towards plastid surfaces is root-cap specific and provides the evidence for actomyosin involvement in root osmosensing. Funct. Plant Biol. 32, 721-736. doi: 10.1071/FP05004

Wu, Y., Yan, J., Zhang, R., Qu, X., Ren, S., Chen, N., et al. (2010). Arabidopsis FIMBRIN5, an actin bundling factor, is required for pollen germination and pollen tube growth. Plant Cell 22, 3745-3763. doi: 10.1105/tpc.110.080283

Yang, W., Ren, S., Zhang, X., Gao, M., Ye, S., Qi, Y., et al. (2011). Bent uppermost internode1 encodes the class II formin FH5 crucial for actin organization and rice development. Plant Cell 23, 661-680. doi: 10.1105/ tpc. 110.081802

Ye, J., Zheng, Y., Yan, A., Chen, N., Wang, Z., Huang, S., et al. (2009). Arabidopsis formin 3 directs the formation of actin cables and polarized growth in pollen tubes. Plant Cell 21, 3868-3884. doi: 10.1105/tpc.109.068700

Zhang, Z., Zhang, Y., Tan, H., Wang, Y., Li, G., Liang, W., et al. (2011). Rice morphology determinant encodes the type II formin FH5 and regulates rice morphogenesis. Plant Cell 23, 681-700. doi: 10.1105/tpc.110.081349

Conflict of Interest: The authors declare that the research was conducted in the absence of any commercial or financial relationships that could be construed as a potential conflict of interest.

Copyright (c) 2021 Diao and Huang. This is an open-access article distributed under the terms of the Creative Commons Attribution License (CC BY). The use, distribution or reproduction in other forums is permitted, provided the original author(s) and the copyright owner(s) are credited and that the original publication in this journal is cited, in accordance with accepted academic practice. No use, distribution or reproduction is permitted which does not comply with these terms. 